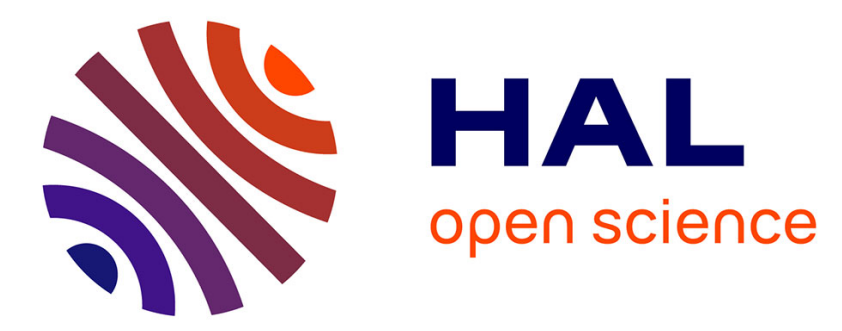

\title{
Using robots for prototyping autonomous intersection management: feasibility and feedbacks.
}

\author{
Abdeljalil Abbas-Turki, Mourad Ahmane, Fei Gao, Wu Jia, Abdellah \\ El-Moudni, Miraoui Abdellatif
}

\section{- To cite this version:}

Abdeljalil Abbas-Turki, Mourad Ahmane, Fei Gao, Wu Jia, Abdellah El-Moudni, et al.. Using robots for prototyping autonomous intersection management: feasibility and feedbacks.. 1st IFAC Conference on Embedded Systems, Computational Intelligence and Telematics in Control, CESCIT 2012, Apr 2012, Würzburg, Germany. pp.308-313. hal-00808564

\section{HAL Id: hal-00808564 https://hal.inria.fr/hal-00808564}

Submitted on 5 Apr 2013

HAL is a multi-disciplinary open access archive for the deposit and dissemination of scientific research documents, whether they are published or not. The documents may come from teaching and research institutions in France or abroad, or from public or private research centers.
L'archive ouverte pluridisciplinaire HAL, est destinée au dépôt et à la diffusion de documents scientifiques de niveau recherche, publiés ou non, émanant des établissements d'enseignement et de recherche français ou étrangers, des laboratoires publics ou privés. 


\title{
Using Robots for Prototyping Autonomous Intersection Management: Feasibility and Feedbacks
}

\author{
Abdeljalil Abbas-Turki, Mourad Ahmane, Fei Gao, Jia Wu, \\ Abdellah El-Moudni, Abdellatif Miraoui \\ Laboratoire Systèmes et Transport, Université de Technologie de \\ Belfort Montbéliard, 90010 Belfort Cedex, France \\ (e-mail: prenom.nom@utbm.fr).
}

\begin{abstract}
This paper presents a protocol of an Autonomous Intersection Management. This protocol is mainly based on the wireless communication between robots and the controller. It allows the robots to cooperatively access to the intersection. It is also a step towards a new way of controlling the ordinary traffic at intersections. The right of way is addressed to each robot individually, according to an optimized sequence. This protocol is tested through a prototype where the robots are safely directed through the intersection. The approach raises some issues related to the wireless communication. These issues are discussed in this paper and a robust protocol is given.
\end{abstract}

Keywords: Autonomous Intersection Management, Cooperative driving, Robots, Wireless communication.

\section{INTRODUCTION}

A fully autonomous vehicle can safely traverse the intersection if it accurately observes its surrounding environment, as shown in DARPA Urban Challenge [Urmson and al. (2008)]. However, such a technology does not respond to the issue of the efficiency of the intersection management. Until today, the optimizations of traffic light split and offset have been the main answer to this issue [Crisman and al. (1993), Takizawa and al. (1998)] and positioning systems has enabled the emergence of new traffic control concepts, recently called Autonomous Intersection Management (AIM). These concepts are mainly supported by:

(1) Positioning technology,

(2) Vehicle-to-vehicle (V2V) and vehicle-to-infrastructure (V2I) communications.

AIM brings the following several novelties:

- Unlike the traditional control systems that decide the duration of phases (the green light given to movements) [Tsugawa (2002)] or decide whether or not the phase is still kept for another period of time [NHTSA (2005), Hunt (1982)], AIM allows to precisely decide which vehicle has the right of way and addresses the right of way to only admitted vehicles.

- The decision is performed according to the actually observed traffic instead of predicting the volume of the traffic from the past statements of the inductive loops.

- It uses cooperative driving techniques for deciding an optimized sequence according to a given criteria.

- It facilitates the deployment of priority policies between vehicles because each vehicle is considered individually.
The scope of this paper is to use robots instead of vehicles in order to prototype an AIM. Since the robots know their positions and their destinations, they can wirelessly negotiate the rights of way with the other robots. We present an AIM prototype that involves more than one robot. This prototype is used to propose a realizable protocol and to prove the feasibility of AIM for industrial application.

AIM allows scheduling the sequences of robots, for industrial applications. Otherwise say, AIM explicitly determines the sequences of robots through a real-time application. A sequence is a stringent order of distributing the rights of way to the robots. Each robot that approaches the intersection is managed separately and waits for receiving its right of way before crossing the intersection. The robot informs the others robots and the intersection manager about its arrival time, its access time and its exit time. Other information such as the movement, the braking, and the speed of the robot could be collected in order to distribute the right of way. AIM requires at least a wireless communication system between the robots that know their positions and the intersection manager.

Moreover, many simulations show that AIM outperforms traffic lights, for urban applications [Gartner (1983), Farges and al. (1983)]. So, the expected advantages of AIM raise at least the issue of the feasibility. However, for safety reasons, realize an intersection without traffic lights, priority or stop signs (roadside signalization) requires a first validation of the protocol through a prototype. Moreover, a successful experience of an intersection of mini-robots opens the opportunity to apply AIM to intersections of vehicles into workshops for improving the traffic efficiency. 
The success of AIM depends on the ability of the proposed protocol to face real-world disturbances, such as the communication times, message loss, and so on. Indeed, on the one hand, the devices and standards involved in cooperative mini robots into a workshop are different from the ones required for the safety applications of cooperative vehicles. Moreover, some additional communication costs are to be considered in the future such as secure communication algorithms (authentication of vehicles) and the reaction time of the subsystems.

This paper is organized as follows. Firstly, it gives a brief literature overview. Then, the prototype of the intersection is presented and the performance of the NXT wireless communication is evaluated. Also, it presents the protocol after discussing the alternatives. Afterward, it describes the conditions of the tests and reports different feedbacks.

\section{A BRIEF LITERATURE OVERVIEW}

AIM can be dated before the last decade. However, to the best of our knowledge, the proposed protocols are limited to particular applications. For instance, in [Van Middlesworth and al., (2008), Dresner and Stone (2008)], the map is split into segments. The allocation of each segment is limited to at most one vehicle.

In 2004, the studies of [Neuendorf and Bruns (2004)] simultaneously with the ones of [Dresner and Stone (2004) have taken a comprehensive step toward realizing the vision of AIM for a wide scale. In both papers, vehicles are fully autonomous but the respective protocols are different. In the first paper, the autonomous intersection is a vehicle platoon controller in a decentralized autonomous intersection management. More precisely, each vehicle directly negotiates with the other vehicles the right of way and adapts its speed to form a platoon with the other vehicles. The protocol has been tested by using few mini robots [Grunewald and al. (2006)]. A Petri net model of the intersection has been proposed. The tests have shown that $3 \%$ of the total passes have resulted on collisions because of the saturation of the communication bandwidth. Without communication, $22 \%$ of the total intersection passes has resulted in collision. Hence, this first experience has shown that the proposed decentralized protocol cannot face the deficiencies of the wireless communication.

The second protocol is theoretically based on the multiagent paradigm. An intelligent agent, called intersection manager, assigns a reservation of space and time to each autonomous vehicle controlled by a driver agent. So, the protocol is centralized. A First Come First Served (FCFS) policy is used for directing vehicles through the intersection. The simulations presented by [Dresner and Stone (2004)] have shown that the protocol of AIM has outperformed the traditional traffic signals. More recently, in [Quinlan and al. (2010)], a mixed reality platform on which an autonomous vehicle can interact with multiple virtual vehicles is implemented. This mixed reality platform confirms the results of the simulations. However, the robots are not as precisely controllable as has been assumed in previous studies. The authors have proposed a buffer time to overcome this drawback. Nevertheless, as stated by the authors, the buffer time is a compromise between the safety and the efficiency. In other words,

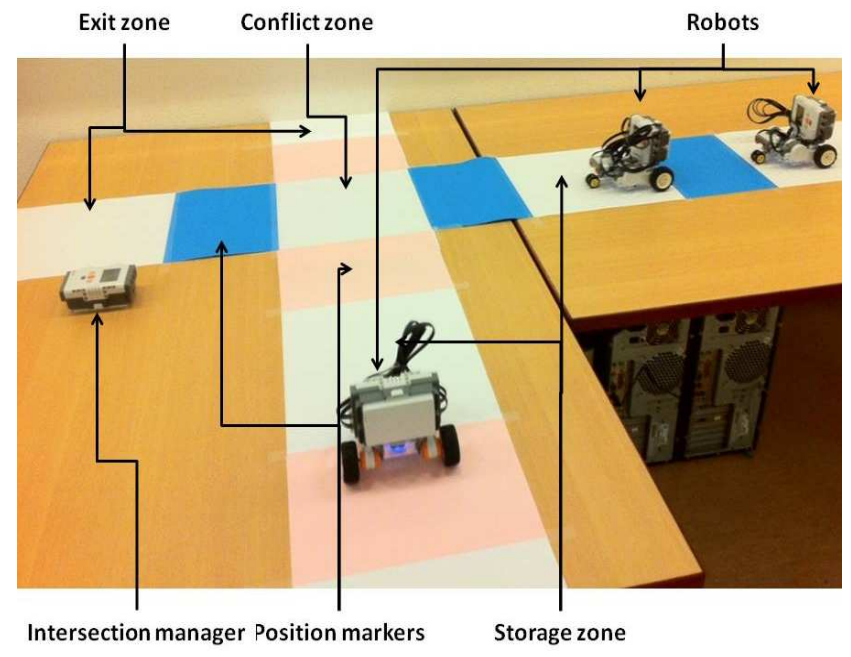

Fig. 1. Prototype of an elementary intersection system

collisions are possible if the buffer time does not match the actual communication delay.

Other intersection management protocols based on wireless communications and positioning technologies have been proposed, simulated and tested. However, they do not match the AIM concept because the signalization system is based on the traffic lights. Indeed, the two AIM protocols share the following important ambitions:

- The accesses to the intersection are ordered (sequence) according to the requests received from the vehicles via the wireless communication.

- Each vehicle receives its right of way individually, with respect to the sequence.

- There is no preprogrammed sequence.

- Regardless technological and practical limitations, such the road capacity or the restriction of the wireless communication, the protocol of the traffic management at intersections is able to consider an unbounded number of vehicles without a preprogrammed schedule.

In the reminder of this paper, we will present a prototype of a protocol that fulfils the AIM's ambitions by using robots.

\section{PROTOTYPE}

The prototype is an elementary intersection composed of two one way lanes, an intersection manager and robots that have to safely traverse the intersection (see Fig.1).

The intersection splits each lane into three zones: a storage zone, a conflict zone and an exit zone. Since the robots must be able to estimate their positions, three position markers are added to each lane. The first one initializes the position given by the robot's odometer. The second one aims to avoid collision and the third one makes the succession of the right of way more efficient. Each position marker is a colored rectangle, as shown in Fig.1. Two colors are used in order to distinguish lanes, i.e. blue for lane 1 and pink for lane 2 .

The intersection manager is a NXT micro-computer. The robots are equipped with one NXT micro-computer, one 

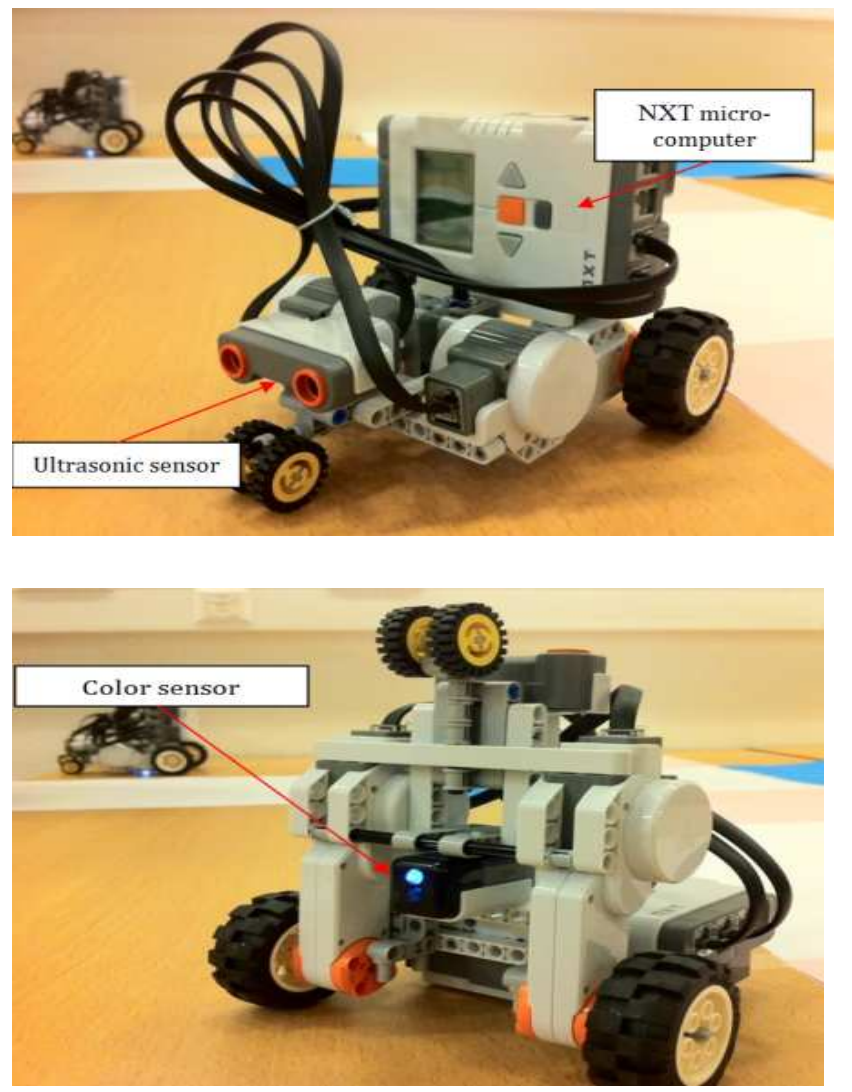

Fig. 2. Robot: Front view and Bottom view

color sensor to recognize the position markers, two interactive servomotors with built-in rotation sensors and one ultrasonic sensor that detects the frontal distance (Fig. 2).

The ultrasonic sensor avoids collision with the precedent robot that moves on the same lane. More precisely, each robot adapts its speed if the frontal distance is lesser than $40 \mathrm{~cm}$. Under this condition, the robot considers itself as a follower [Gipps (1981)].

\subsection{Wireless Communication}

The Bluetooth system on the NXT is designed to run in a master/slave configuration. In other words, three or more NXT micro-computers can only communicate together under a centralized architecture. Hence, each robot cannot directly communicate with the other robots. The intersection manager is chosen to be the node of the wireless network (master). The wireless communication is used to support all the communications between each pair of robots (see Fig. 3).

The negotiation through a centralized architecture is stated by the protocol presented by [Dresner and Stone (2004)]. As discussed hereafter, the centralized architecture is a first step towards a safer autonomous intersection. So, we will not consider this architecture as an obstacle to our ambition.

\subsection{NXT Bluetooth}

NXT Bluetooth enables to build an autonomous intersection. However, the success of an autonomous intersection

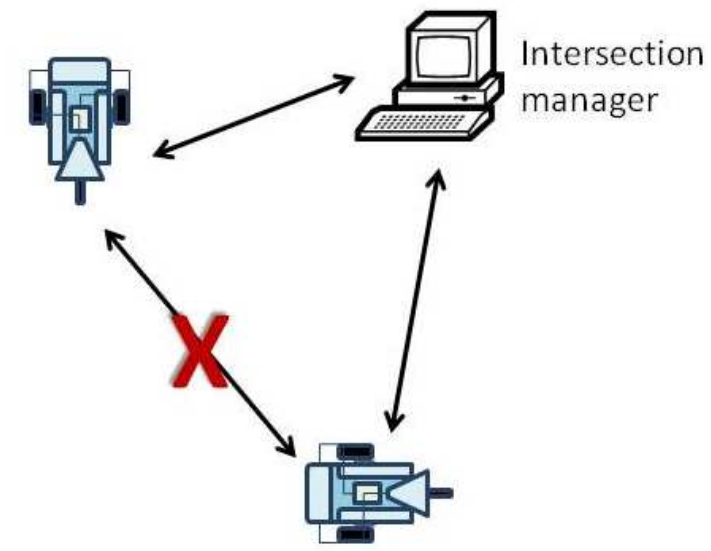

Fig. 3. Communication between two robots

depends on whether the proposed protocol is able to face the wireless disturbances. So, we deeply assess the reliability of NXT Bluetooth. Four parameters are estimated and optimized: the ratio of successful messages, the average time between two successful messages, the communication time and the percentage of unordered messages. But first, let us draw the reader attention to the "order matter" and to the "cycle time" issue.

1) Order matter. NXT Bluetooth conserves the order of the messages received from the same NXT microcomputer. However, the "whole message order" could be not respected, since there are more than one message source.

The NXT master can only communicate with one slave source at a time. For example, if the intersection manager is communicating with robot 2 it must finish sending or receiving data before it can communicate with robot 3 . Thus, the intersection manager receives messages from robot 1 , from robot 2 and after from robot 3 . This allows a better order of the received messages. However, it partially resolves the whole order matter. For instance, if robot 1 sent a request of the right of way before robot 3 , the request received from robot 3 can be treated before, since:

- The first request of robot 1 is missed.

- The request of robot 1 is sent when the intersection manager is hearing messages from robot 2 .

This order matter raises the problem of deadlock that will be discussed in Subsection 5.3.

2) Cycle time. Each program is a recurring cycle because of the dynamic nature of the application. The duration of the cycle depends on the compiled algorithms and on the processor. The NXT micro-computers empirically limit our investigation to a cycle time greater or equal to $50 \mathrm{~ms}$.

3) Tests. The test begins by synchronizing the clock of NXT micro-computers, using touch sensors. Then, messages of four digits are sends periodically from the three robots to the intersection manager as well as from the intersection manager to all robots. The four digits indicate the time and the origin of the message. A test covers 90s during which each NXT micro-computer logs the sent messages and the received messages. Afterward, all log 


\begin{tabular}{|l|}
\hline Controller to All targets: \\
Messages status: \\
Attempt send: 991 \\
Success send: 290 \\
Ratio: $29.26 \%$ \\
Messages communication delay: \\
Average: $230.80 \mathrm{~ms}$ \\
Min: $31 \mathrm{~ms}$ \\
Max: $402 \mathrm{~ms}$ \\
Std deviation: 93.07 \\
Success messages time interval: \\
Average: $918.88 \mathrm{~ms}$ \\
Min: $272 \mathrm{~ms}$ \\
Max: $6825 \mathrm{~ms}$ \\
Std deviation: 752.44 \\
\hline
\end{tabular}

\begin{tabular}{|l|}
\hline All targets to Controller: \\
Messages status: \\
Attempt send: 1806 \\
Success send: 138 \\
Ratio: $7.64 \%$ \\
Messages communication delay: \\
Average: $785.12 \mathrm{~ms}$ \\
Min: $674 \mathrm{~ms}$ \\
Max: $1097 \mathrm{~ms}$ \\
Std deviation: 74.99 \\
\\
Success messages time interval: \\
Average: $1945.56 \mathrm{~ms}$ \\
Min: $750 \mathrm{~ms}$ \\
Max: $7350 \mathrm{~ms}$ \\
Std deviation: 1313.81 \\
\hline
\end{tabular}

Fig. 4. Global statistics: Robot's cycle time is $150 \mathrm{~ms}$. Intersection manager's cycle time is $90 \mathrm{~ms}$
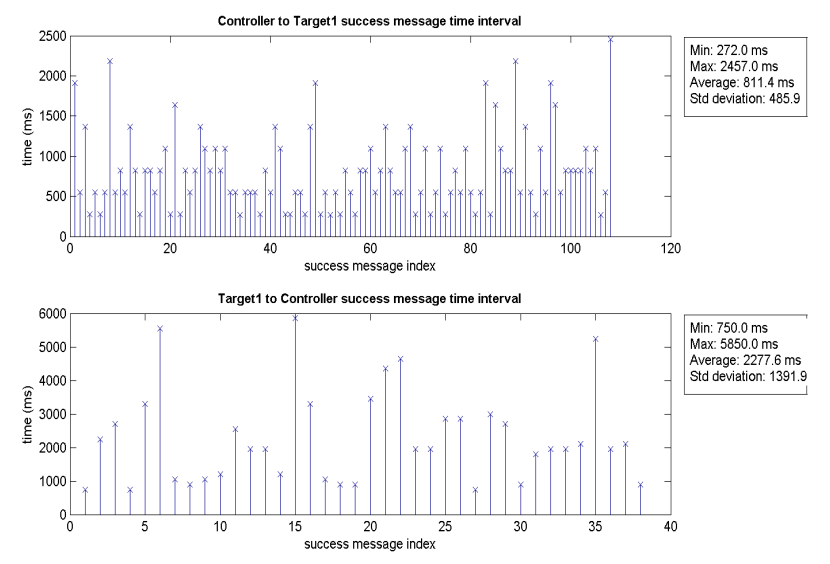

Fig. 5. Results of the communication between robot 1 and the intersection manager during $90 \mathrm{~s}$

files are compared. Twenty combinations of cycle times are tested ten times. The cycle times vary from $50 \mathrm{~ms}$ to $900 \mathrm{~ms}$. The optimal result is empirically obtained. Each robot sends a message every $90 \mathrm{~ms}$ and the intersection manager sends a message every $150 \mathrm{~ms}$. Results of this combination are presented in Fig. 4 and Fig. 5.

From Fig. 4 and Fig. 5, we can note that the performances of messages sent by the robots are low. Indeed, the communication delay is about $2 \mathrm{~s}$ and the ratio of lost messages is high. These low performances are an opportunity to assess whether the proposed protocol can face the communication problems. Indeed, the NXT Bluetooth brings us face-toface with some of the most important questions of the feasibility of AIM. The answers to these questions allow us to build a robust protocol.

\section{PROTOCOL}

Let us first draw the reader attention to the safety issue. The decentralized prototype presented by [Grünewald and al. (2006)] has shown that collisions would occur if a robot had missed some messages broadcasted by another robot. This is due to the fact that each robot autonomously decides for itself according to the owned presence list but this list can be inconsistent. A centralized architecture overcomes this problem, if the decision is taken by a referee that owns a common presence list. This architecture contributes to avoid some collisions, even if the list is incomplete. Indeed, all missed robots are not able to traverse the intersection until they are included into the presence list of the intersection manager.

There are at least two strategies to allow a robot to go through the intersection. The simplest one informs a particular robot that the conflict zone is free. A more sophisticated one specifies the time and the velocity of the reservation given to each robot. The second strategy deserves more attention. This strategy states that the robot loses the reservation if it determines that it will not arrive at the intersection at the time and/or the velocity specified in the reservation. In this protocol, the main burden of avoiding deadlock falls on the communication efficiency. Indeed, on the one hand, a robot that misestimates the access time will delays all the following robots until it obtains a satisfactory access time. At the same time, the delayed robots have to negotiate a new access time. On the other hand, if the communication delay is high because of several simultaneous new requests, the proposed access time can be unfeasible.

The proposed protocol is as follows (see Fig. 6). The system of right of way is a kind of a padlock. If the padlock is unlocked (open), the robot has the right of way. Otherwise, the robot is not allowed to traverse the conflict zone and thus has to brake before the end of the storage zone. Hence, the right of way is a key that the robot receives in order to unlock the received padlock. This key is owned by the intersection manager. When a robot arrives at the intersection, it passes over the first position marker. The infrastructure sends the map of the intersection and a locked padlock. Then, it computes the best sequence and accordingly distributes the key to first admitted platoon of robots that moves on the same lane. When the last admitted robot passes over the third position marker, it sends the last distributed key to the infrastructure manager whom proceeds to free robots of the opposite lane and so on. If some robots arrive before the end of the sequence, the intersection computes a new sequence without considering robots that have already received the key, even if they have not yet leaved the storage zone. The objective of this rule is to avoid an emergency braking to robots that have already received the key. If the new arriving robot comes from the lane on which the already authorized robots move, it can immediately receive the key, according to the adopted strategy for sequencing robots.

\section{TESTS}

The proposed protocol has been tested through two policies for directing robots through the intersection. Each policy was implemented by LabView.

The first one is the FCFS policy and the second one is an adaptation of the algorithm proposed in [Wu and al. (2007)]. Indeed, the algorithm is designed for an intersection with traffic lights and it is quite greedy. However, the algorithm is based on a Petri Net model that fits the objective of a personalized signalization. The algorithm aims to empty the intersection as soon as possible. The 


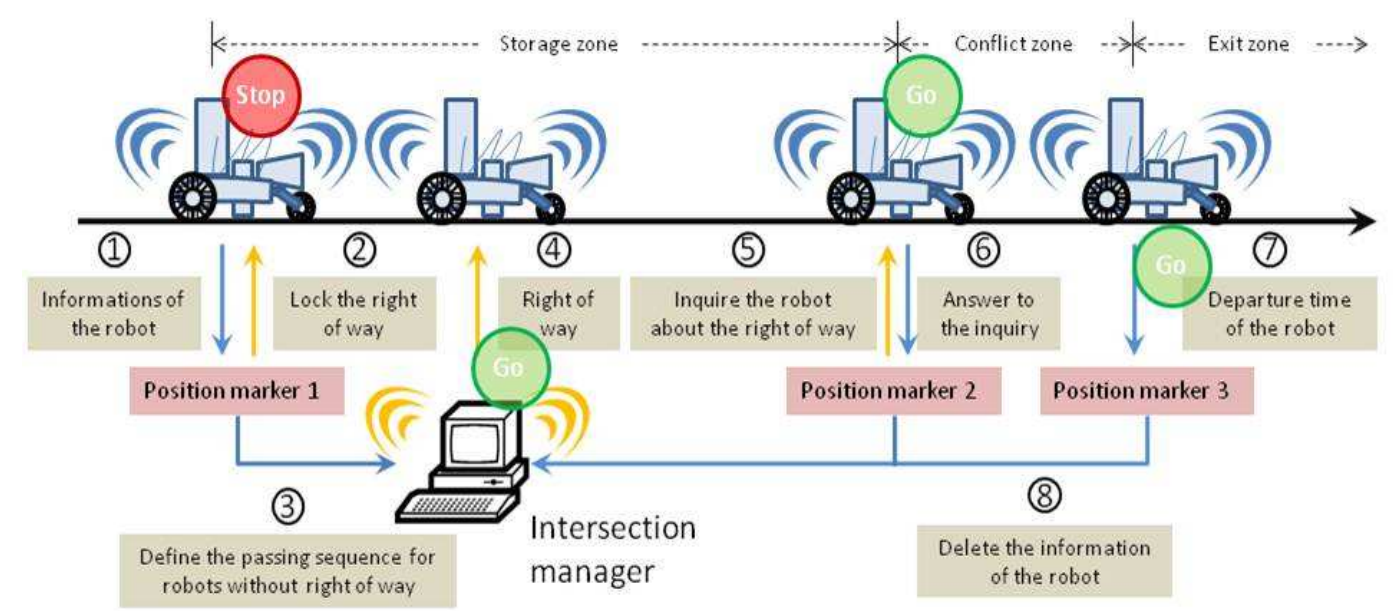

Fig. 6. The Protocol
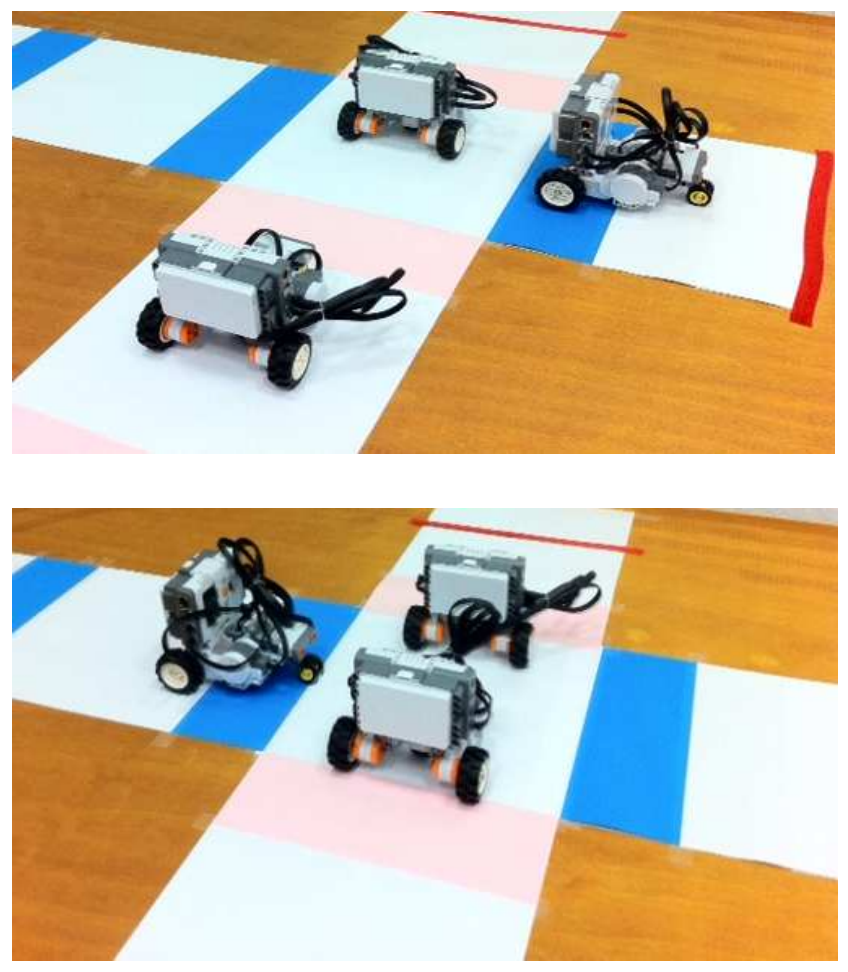

\subsection{Safety Issue}

During the laboratory tests, we have not observed any collision. However, the "Journée portes ouvertes à l'UTBM" has revealed a collision risk thanks to public manipulation of the robots. Indeed, collision has been caused by an indelicate manipulation that has changed the position of the color sensor. So, the deficient robot has not detected the positions markers. This risk must be deeply studied if we would implement an autonomous intersection with high speed of robots. Indeed, the robustness of the protocol depends also on the used positioning system and on its protection. We can also design a redundant positioning system. However, the scale of our prototype does not allow us to push further the investigation. Nevertheless, tests have proven that the protocol faces the weak performances of the wireless communication, without collisions.

\subsection{Efficiency Issue}

The first tests of FCFS have shown that the sequence could be obviously optimized if three robots would traverse the intersection. This is the reason why we have implemented WAE.

So, let us introduce this configuration of the intersection. The maximal speed of each robot is limited to $0.064 \mathrm{~m} / \mathrm{s}$ and the length of the intersection is $0.64 \mathrm{~m}$. Without signalling system, the travel time of each robot is then

Table 1. Results of tests

been simplot detects the frontal distance. This policy will be called WAE (formed by the initials of authors), in the rest of the paper.

Many tests of both policies have been done. A test takes at most 30s. Each policy has been tested 200 times in the laboratory. The robots have been randomly distributed and started (see Fig. 7).

Moreover, the prototype has been exposed to public, in different events. The results of all tests are presented in Table1. The most important one was: "Journée portes ouvertes à l'UTBM", where a public with a wide range of age and of scholar level has manipulated the prototype and has tested several scenarios, according to their curiosity. This exposition has been rich in terms of feedbacks.

\begin{tabular}{|l|c|c|c|}
\hline & Collision & $\begin{array}{c}\text { Resolved } \\
\text { deadlock }\end{array}$ & Deadlock \\
\hline $\begin{array}{l}\text { Low performance } \\
\text { of wireless } \\
\text { communication }\end{array}$ & $0 \%$ & $0.5 \%$ & $0 \%$ \\
\hline $\begin{array}{l}\text { Without } \\
\text { communication }\end{array}$ & $0 \%$ & $0 \%$ & $100 \%$ \\
\hline $\begin{array}{l}\text { One robot with an } \\
\text { erroneous position } \\
\text { (a robot that do } \\
\text { notdetect that is in } \\
\text { the storage zone) }\end{array}$ & $37.2 \%$ & $0 \%$ & $\begin{array}{c}37.2 \% \\
\text { (due to } \\
\text { collision) }\end{array}$ \\
\hline
\end{tabular}


10s. The comparisons of both scenarios have revealed that the delay can be reduced by $5 \mathrm{~s}$.

Regardless the chosen policy for directing robots through the intersection, the communication time can decrease the efficiency of the intersection. Indeed, this time delays the key distribution to the robots that move on the opposite lane. The average time of this delay is about $1 \mathrm{~s}$.

\subsection{Deadlock Issue}

We have met one deadlock situation for all tests (more than 400). This low number of deadlock situation is due to the NXT Bluetooth explained in Subsection 3.2 "order matter".

The deadlock situation was studied before the implementation. The centralized protocol is the cause of this problem. Indeed, the order of the received requests can be different from the order of robot's arrivals. For example, robot 2 arrives first and robot 1 arrives later. Both robots move on the same lane. Due to missed messages, robot 2 is considered by the intersection manager as it is in the second position. Robot 1 receives the right of way, but robot 2 is stopped.

The adopted solution to this problem consists in using the ultrasonic sensor. If a robot with an unlocked right of way is stopped by a robot in front of him, the intersection manager distributes the key to robots that share the same lane and $\log$ the event. We have manually simulated overtaking between robots, in order to generate the combinations of the deadlock situations. All manually deadlocks are reported by the intersection manager and resolved with success.

\section{CONCLUSION AND FUTURE WORKS}

This paper has presented the use of mini robots to test the feasibility of the concept of the autonomous intersection management. The concept is based on the wireless communication and on the positioning technology. The presented prototype is based on NXT technology. Low performance of the provided wireless communication is observed, with regard to the ambition of a real time application. A robust protocol is proposed to face such a problem. This protocol is open to the implementation of different scheduling algorithms. Two algorithms are tested. The prototype shows that, the protocol could be deployed for industrial applications, under the condition of providing a robust positioning system.

Several issues deserve further investigations. The first issue consists in to extending the proposed protocol to ordinary vehicles, by using the onboard signalization. The second one is dedicated to the scheduling algorithms.

\section{REFERENCES}

[Crisman and Thorpe (1993)] Crisman, J.D., and Thorpe, C.E. (1993). SCARF: a color vision system that tracks roads and intersections. IEEE Trans. Robotics and Automation, volume 9, issue 1, pages 49-58.

[Crisman and Thorpe (1993)] Dresner, K., and Stone, P. (2004). Multiagent Traffic Management: A Reservation-Based Intersection Control Mechanism.
In the 3rd Int. Joint Conf. on Autonomous Agents and Multiagent Systems, pages 530-537.

[Crisman and Thorpe (1993)] Dresner, K., and Stone, P. (2008). Mitigating Catastrophic Failure at Intersections of Autonomous Vehicles. In The Fifth Workshop Agents in Traffic and Transportation (ATT 08). Estoril, Portugal.

[Crisman and Thorpe (1993)] Farges, J.L., Henry, J.J., and Tufal, J. (1983). The PRODYN real-time traffic algorithm. In 4th IFAC Symposium on Transportation Systems, Baden Baden, Germany.

[Crisman and Thorpe (1993)] Gartner, N. (1983). OPAC: A Demand-Responsive strategy for traffic signal control. Transportation Research Record, volume 906, pages $75-81$.

[Crisman and Thorpe (1993)] Gipps, P.G. (1981). A behavioural car-following model for computer simulation. Transportation Research Part B: Methodological, volume 15 , issue 2 , pages $105-111$.

[Crisman and Thorpe (1993)] Grünewald, M., Rust, C., and Witkowski, U. (2006). Using mini robots for prototyping intersection management of vehicles. In Proceedings of the 3rd International Symposium on Autonomous Minirobots for research and edutainment, pages 288-294.

[Crisman and Thorpe (1993)] Hunt, P.B. (1982). The SCOOT on-line traffic signal optimisatoin technique. Traffic Engineering and Control, volume 23, pages 190-192.

[Crisman and Thorpe (1993)] Neuendorf, N., Bruns, T. (2004). The vehicle platoon controller in the decentralised, autonomous intersection management of vehicles. Mechatronics, 2004. In Proceedings of the IEEE International Conference on, pages 375-380.

[Crisman and Thorpe (1993)] NHTSA, (2005). Vehicle Safety Communications Project Task 3 Final ReportIdentify Intelligent Vehicle Safety Applications. Enabled by DSRC, U.S. Department of Transportation: National Highway Traffic Safety Administration. Washington, USA.

[Crisman and Thorpe (1993)] Quinlan, M., Tsz-Chiu, A., Zhu, J., Stiurca, N., and Stone, P. (2010). Bringing simulation to life: A mixed reality autonomous intersection. IROS, IEEE/RSJ International Conference on.

[Crisman and Thorpe (1993)] Takizawa, H. , Shirai, Y. , Miura, J. , Kuno, Y. (1998). Planning of observation and motion for interpretation of road intersection scenes considering uncertainty. Proceedings of Intelligent Robots and Systems, pp. 520-525.

[Crisman and Thorpe (1993)] Tsugawa, S. (2002). Intervehicle communications and their applications to intelligent vehicles: an overview. Proceedings of IEEE Intelligent Vehicle Symposium, Versailles, France.

[Crisman and Thorpe (1993)] Urmson, C. and al. (2008). Autonomous driving in urban environments: Boss and the Urban Challenge. Journal of Field Robotics, volume 25 , issue 8 , pages $425-466$.

[Crisman and Thorpe (1993)] Van Middlesworth, M., Dresner, K., and Stone., P. (2008). Replacing the Stop Sign: Unmanaged Intersection Control for Autonomous Vehicles. In Proceedings of International Conference on Autonomous Agents and Multiagent Systems, Portugal. 
[Crisman and Thorpe (1993)] Wu, J., Abbas-Turki, A., Corréia, A., and EL Moudni, A. (2007). Discrete Intersection Signal Controling. In IEEE International Conference on Service Operations and Logistics, and Informatics, pages 27-29. 